\title{
Efficient Forward Dynamics Simulation and Optimization of Human Body Dynamics
}

\author{
Maximilian Stelzer* and Oskar von Stryk \\ Simulation and Systems Optimization Group, Technische Universität Darmstadt \\ Hochschulstrasse 10, D-64289 Darmstadt
}

Key words Forward dynamics simulation, forward dynamics optimization, multibody systems, optimal control, biomechanics, human motion.

\begin{abstract}
The modeling of the time dependent, dynamic behavior of the human musculoskeletal system results in a large scale mechanical multibody system. This consists of submodels for the skeleton, wobbling masses, muscles and tendons as redundant actuators. Optimization models are required for the simulation of the muscle groups involved in a motion. In contrast to the inverse dynamics simulation the forward dynamics simulation enables to consider very general problem statements in principle. The paper presents a new approach to the forward dynamics simulation and optimization of human body dynamics which overcomes the enormous computational cost of current approaches for solving the resulting optimal control problems. The presented approach is based on a suitable modeling of the dynamics of the musculoskeletal system in combination with a tailored direct collocation method for optimal control. First numerical results for a human kick demonstrate an improvement in computational time of two orders of magnitude when compared to standard methods.
\end{abstract}

\section{Introduction}

The kinetic analysis of a measured human motion as well as the generation of an optimal goal oriented human motion both lead to the problem of finding suitable activations of the muscles involved. This paper presents first steps of the integrated development of new methods for efficient modular and object oriented kinetic modeling and also for simulation and control of the human muscle-skeleton-apparatus with optimal control methods. The overall aim is to solve forward dynamics simulation for investigation of dynamic human motions involving many muscle groups, the treatment of general muscle models and general objective functions for the control of redundant muscle groups with a much higher efficiency than currently possible. New methods with higher order of efficiency can pave the way for completely new types of investigations in ergonomics, medicine and biology.

The paper is structured as follows. Section 2 states the problem and gives a brief survey of current literature. In Section 3 dynamics algorithms for the human body are described. Section 4 introduces our new approach to solving the forward dynamics simulation and optimization problem. First numerical results are given in Section 5. The paper concludes with Section 6.

\section{Simulation and Optimization of Dynamic Biomechanic Motions - State of the Art}

\subsection{Problem Statement}

In biomechanical systems, redundancies occur in two different ways: First, one overall motion of legs and/or arms from an initial to a final position generally may be performed by an infinite number of joint angle trajectories; second, as human joints are actuated by redundant muscle groups a specific kinematic joint angle trajectory may be realized by an infinite number of different activations of the muscles involved. The central problem statement addressed in this paper is as follows: Find the activations $\boldsymbol{u}(t)=\left(u_{1}(t), \ldots, u_{n_{m}}(t)\right)^{T}$ of each of the $n_{m}$ muscles involved so that the resulting calcium ion concentration $\gamma_{i}$ caused by the activation $u_{i}$ of each muscle $i$ leads to forces $F_{i}, i=1, \ldots, n_{m}$, which cause a motion of all $n_{q}$ joints (i.e. joint angle trajectories $\boldsymbol{q}(t)=\left(q_{1}(t), \ldots, q_{n_{q}}(t)\right)^{T}, 0 \leq t \leq t_{f}$ ) which

\footnotetext{
* Corresponding author, e-mail: stelzer@sim.tu-darmstadt.de, Phone: +496151 164722, Fax: +496151 166648
} 
1. is equal or as "close" as possible to the kinematic and/or kinetic data of a human body motion measured in experiments (inverse problem), or

2. best fulfills some motion goal like maximum jump height or width or fastest possible walking or running (forward problem).

While in the first case only the redundancy of the muscles actuating one joint must be considered, the second case incorporates also the additional level of redundancy with respect to the overall movement. "Close" in the first case may be measured by an objective function, e.g. the integral over the difference of measured and calculated joint angle trajectories. The goal achievement in the second case can be measured as well by a suitable objective function as time or energy required.

Accurate and efficient numerical investigation of the forward problem in case of the dynamic behavior of large parts of or even the complete human body, consisting of coupled submodels for skeleton, wobbling masses, muscles and tendons and the control mechanisms of the redundant muscle groups involved in a movement, is yet not satisfyingly solved. Kinetic modeling of the muscle-skeleton-apparatus leads to very large systems of differential equations. Usually a large number of controls results from the many redundant muscle groups involved. Moreover, several different hypotheses on suitable objectives and constraints exist for determining the controls of each single muscle involved by simulation and optimization.

Therefore, forward dynamics simulation of a human motion leads to high dimensional, nonlinear optimal control problems. Current approaches even for problems with reduced models of the whole human body require computation times of days or weeks on workstations, cf. [2]. Forward dynamics simulation based on a validated dynamics model and model parameters has the important potential of predicting certain motions. While forward dynamics simulation is state of the art in vehicle and robot dynamics, e.g. [25, 28, 52, 53], it is still at an early stage in the area of human motion.

On the other hand, inverse dynamics simulation investigates given kinematic position and velocity trajectories of a human motion (e.g. by measurement). Together with appropriate modeling approaches it allows a comparatively fast numerical calculation of the controls of each muscle group if very restrictive assumptions on the underlying model like special objective functions for control of the muscles involved are made. Inverse dynamics simulation for a measured human motion gives an interpretation of the acting forces and torques on the level of the single muscles involved.

\subsection{Dynamics Modeling}

Modeling of the dynamics of human motion involves mainly the following three components of motion generation: (i) skeleton and wobbling masses as a mechanical multibody system (MBS), (ii) muscles and tendons as the (redundant) actuators of the system with inherent dynamic behavior, (iii) control concepts for the activation of the muscle groups involved in generating the motion.

\subsubsection{Multibody Dynamics Modeling of Skeleton and Wobbling Masses}

Several methods and programs for modeling and simulation of the dynamics of general multibody systems of various structures exist which are in principle also applicable to the dynamic modeling of the human motion apparatus, for modeling walking or grasping motion of parts of or the whole human body, e.g. ADAMS, DADS or SIMPACK [52, 53].

The assumptions underlying these general-purpose methods do usually not allow to exploit special structure in MBS. E.g. a standard formulation of MBS with constraints is the descriptor form resulting in a possibly large system of differential algebraic equations (DAEs) of index 3 [52]. By exploitation of special properties of the MBS, e.g. a smaller system of ordinary differential equations (ODEs) may be obtained which can numerically be solved more robustly and efficiently. Furthermore, only few general purpose tools for MBS modeling and simulation are prepared for the numerical solution of an optimal control problem of the redundant muscle groups involved in a motion.

On the other hand, for four-legged and bipedal walking robots efficient methods for modeling of the robot dynamics have been established in recent years. Dynamic motion behavior of walking robots is characterized by a high number of degrees of freedom and many actuated joints and a tree structured MBS with switching contact situations. Recursive methods like [10, 33] are especially well suited for MBS with a large number of degrees of freedom. For tree structured MBS with constraints and inverse kinematics models (like four-legged or bipedal walking robots) modeling of the constraints by DAEs may be transformed using a reduced dynamics approach to a numerically more efficient and robust solvable system of fewer ODEs [25]. It is therefore worth to investigate the extension from modeling the dynamics of humanoid robots [32] to human body dynamics.

\subsubsection{Dynamics Modeling of Muscles and Tendons}

For modeling of the dynamic motion and force behavior of muscles as contracting actuators with serial and parallel elasticities and active contractile elements a number of well investigated models have been developed. They describe the muscle forces in relation to muscle length, muscle velocity and muscle activation as the many models based on the 
fundamental approaches of Hill and Huxley, cf. [43, 45]. Almost all models from literature assume that the muscle forces act at a point. For non-punctual areas of force application the muscles are divided into several muscles with single points of actuation. Several approaches exist for modeling the muscle paths as the straight line method (modeling the muscle path to connect the points of application in a straight line), the centroid line method (modeling the muscle path to connect the centers of mass of the muscle cross sectional areas) or the obstacle set method (modeling the muscle path to move freely sliding along the bones). A survey of these approaches may be found for example in [14, 45].

\subsubsection{Control of Redundant Muscle Groups}

Investigation of the real control mechanisms of muscles, that apply to reflexes or controlled motion by the central nervous system, is still a wide open subject of research in neurophysiology. Up to now, only few validated approaches for mathematical models exist. In biomechanics, however, it is a widely accepted hypothesis, that the control of the redundant muscles involved in a motion usually follows some optimality criteria. For different types of motion and different test persons different optimality criteria $\mathcal{J}$ have been suggested, e.g. uniform distribution of the weighted forces $\boldsymbol{F}=\left(F_{1}, \ldots, F_{n_{m}}\right)^{T}$ needed for a certain joint motion to the muscles involved in some $k$-norm, where $k=1,2,3,4$ or $\infty$, see e.g. [43, 50]. The weights are positive characteristics $\boldsymbol{N}=\left(N_{1}, \ldots, N_{n_{m}}\right)^{T}$ of the muscle's capability like cross sectional areas or maximum muscle strength. For $k=\infty$ minimization is performed with respect to the maximum load of the muscles:

$$
\mathcal{J}=\sum_{i=1}^{n_{m}}\left(\frac{F_{i}}{N_{i}}\right)^{k}=\|\boldsymbol{F} . / N\|_{k}^{k}, k \in\{1,2,3,4\} \quad \text { resp. } \quad \mathcal{J}=\left\|\frac{F_{i}}{N_{i}}\right\|_{\infty}=\max _{i}(\boldsymbol{F} . / \boldsymbol{N}) .
$$

Here, $\boldsymbol{F} . / \boldsymbol{N}$ denotes the elementwise quotient like in MATLAB notation. Another approach is to minimize the energy consumed by all muscles, consisting of resting heat, activation heat, maintenance heat, shortening heat, and the mechanical work performed [66].

\subsection{Simulation of Dynamic Motion}

Simulation of time-dependent behavior of a human motion that is modeled according to the previously mentioned details not only means the numerical integration of an ODE or DAE system of large size, but also the solution of a static or dynamic optimization problem for the controls of the redundant muscle groups involved. If a sequence of static frames (snapshots) of a motion is considered, this results in a sequence of static optimization problems. Their solution however is only for very slow motions an acceptable approximation to the solution of the dynamic optimization (i.e. optimal control) problem over the continuous time span of the whole motion, see, e.g. [4, 23].

\subsubsection{Inverse Dynamics Approach}

Inverse dynamics simulation for a given, usually measured, motion obtains the activations for the muscle groups involved under the assumption of certain criteria for solving the redundancy problem. Thus, practically only given motions can be analyzed; predictions of motions that are goal-oriented as optimal reaching of a certain position, jumping as high or far as possible, running as fast or energy-efficient as possible etc. can not or can only very limitedly be obtained, e.g. [8].

Approaches to extend inverse dynamics simulation to the optimization of human motion are based on very special assumptions (like min/max criteria) to the optimality criterion for solving the redundancy problems of the muscles and use a low dimensional parameterization of the free parameter space for being able to numerically solve the resulting optimization problem efficiently, see. e.g. [49, 50]. For slow motions dynamic properties of the wobbling masses do not effect the quality of the solution, and only for slow motions special $\mathrm{min} / \mathrm{max}$ criteria for solving the redundancy problems of the human motion apparatus on the level of muscles and tendons are justified. Distribution of the total forces that act at one joint and of the torques to the muscles then is done according to different parameters of the muscles. But if faster motions shall be investigated, other optimality criteria must be used.

From a biomechanical point of view it is desired not only to investigate fast motion but also to use and evaluate different optimality criteria. Up to now there are no methods to solve these problems with inverse dynamics simulation satisfyingly. First approaches to the efficient treatment of loops that occur due to parallel muscles, may be found in [39]. Inverse dynamics there also is not solved for general optimality criteria. In an approach of two stages first the joint torques and then the muscle forces are calculated.

\subsubsection{Forward Dynamics Approach}

With forward dynamics simulation, on the other hand, both analysis of given motion and calculation and optimization of free motion are possible in principle. Starting from the muscle activations (which are to be determined) forward dynamics 
simulation calculates the resulting motion. Analysis of motion of parts of or even the whole human body is possible with it and leads to a high dimensional nonlinear optimal control problem. Advantageous with the analysis of human motion by forward dynamics simulation and optimization is the fact, that differences of measured and calculated motion may be included in the optimality criterion by an additional term consisting, e.g. of the integral of the square of the deviation. Thus, measurement errors may be compensated for, while with inverse dynamics simulation small measurement errors in the given kinematic trajectories may lead to large errors in the calculated muscle forces.

Numerical optimization using forward dynamics simulation currently most often is treated by application of methods of transforming the optimal control problem by parameterization of the controls (direct shooting) [64] to a finite-dimensional, constrained, nonlinear optimization problem, which is solved by methods of sequential quadratic programming (SQP) type. These approaches are usually not tailored to the problem structure. For the numerical calculation of gradients of the objective function and constraints with respect to the optimization parameters of the control parameterization the sensitivity matrix of the solution of the (ODE or DAE) state differential equations with respect to the optimization parameters has to be calculated [36]. For human motion dynamics this is often done by external numerical differentiation (END) with difference approximation [40, 46, 59]. END is not only computationally very expensive because the differential equations have to be integrated at least as often as the number of grid points in a piecewise polynomial discretization of the controls and, thus, leads to extremely high computation times for motions with a large number of muscle groups. But also additional errors caused by uncoordinated variable step size integration may cancel many if not all valid digits of the gradient approximation. Therefore, so-called internal numerical differentiation (IND) methods are preferable [36] which efficiently and reasonably accurate compute the sensitivity matrix with an extended numerical integration method and using the ODE Jacobian as additional input.

For example the calculation times for vertical jumping motions of a planar leg model with 9 muscle groups $[9,58]$ on a workstation have been reported to be within days [56]. For a spatial model of the whole human body with 54 muscle groups even computation times on workstations in the region of months have been reported [2]. In [5] computation times using a normal computer are compared with those using MIMD parallel and vector parallel computers. The method from [46] is applied to a 14 dof model with 46 muscle tendon groups. Computation times range from one to three months on a normal computer (SGI Iris 4D25), $77 \mathrm{~h}$ on a vector parallel computer and $88 \mathrm{~h}$ on a MIMD parallel computer.

\subsection{Application Scenarios Investigated}

Due to the high computational effort for treating the whole human body, currently only parts of the human body and its interaction with the world are considered, e.g. [2, 18, 26, 41, 42, 68]. In $[6,17,34,40]$ cycling motion is investigated, in [17] to find an optimal cycling machine. In [34] to solve the optimal control problem, the differential equations are not, like commonly done, treated by direct shooting but with a direct collocation approach. A model of a single leg is used for handling a vertical jumping motion in [58]. In [3] a walking motion is optimized. Here, a three dimensional model with 10 segments, 23 dof (including a 6 dof free floating base) and 54 muscle-tendon-units is used. In [22] approaches for foot and muscle modeling for generating stable walking motion have been investigated. Skeletal dynamics, muscle paths, muscle tendon actuators and the relationship between muscle activation and muscle contraction have been examined in [45]. An extended approach to muscle path modeling may be found in [13]. From data of the "Visible Human Male" project [1] and in vivo measurements a dynamical model has been established [14] whose kinematic structure was published in [12]. The necessity of taking into account the special properties of wobbling masses was stated in [21,37]. Approaches to coupling of wobbling masses to the rigid body model of the skeleton may be found in [20, 37, 54]. Properties of 26 muscle groups of shoulder, elbow, and hand joint are presented in [15]. In [47] a three dimensional model of the knee may be found. Geometric data was gained from dead bodies. The contact areas of thigh and tibia are modeled to be deformable, those between thigh and patella to be rigid. 12 elastic elements describe ligaments and capsules; in total 13 muscle-tendon-units are modeled. An optimization is performed not for a complete motion, but for single points of time. Investigation of control concepts, which are supposed to be applied in bipedal walking in nature, have been made in [29].

\section{Dynamics Algorithms for the Human Body}

\subsection{Recursive Multibody Systems Dynamics Algorithm ABA}

General multibody system dynamics is modeled by the well known differential equations

$$
\mathcal{M}(\boldsymbol{q}) \ddot{\boldsymbol{q}}=\boldsymbol{\tau}-\mathcal{C}(\boldsymbol{q}, \dot{\boldsymbol{q}})-\mathcal{G}(\boldsymbol{q})+J_{c}(\boldsymbol{q})^{T} \boldsymbol{f}_{\boldsymbol{c}},
$$

where $\boldsymbol{q}$ are the joint angles, $\boldsymbol{\tau}$ are the total torques, $\mathcal{M}$ is the mass matrix, $\mathcal{C}$ are the Coriolis and centrifugal forces, $\mathcal{G}$ the gravitational forces, and $J_{c}^{T} f_{c}$ the contact forces. For solving these equations, the $\mathcal{O}(N)$ Articulated Body Algorithm (ABA) $[10,51]$ has been shown to be an accurate and numerically stable algorithm superior to the Composite Rigid Body 
Algorithm (CRBA) [28, 38] as it introduces less cancellations of terms [44]. It exploits the linear relationship between accelerations in a rigid-body system and the applied forces; in particular, the definition of the articulated body inertia, the inertia of the 'floppy' outboard chain of rigid bodies not subject to applied forces, has permitted the construction of a recursive algorithm for the forward dynamics [10]. The similarities to the Kalman filtering and smoothing algorithms led to an alternative decomposition of the mass-inertia matrix, which in turn led to an $\mathcal{O}(N)$ closed-form expression for the inverse mass matrix [51] (see also [25] for details):

$$
\begin{aligned}
\mathcal{M} & =H^{T} \phi^{T} M \phi H & & \text { (Newton-Euler Factorization) } \\
\mathcal{M} & =[I+K \phi H]^{T} D[I+K \phi H] & & \text { (Innovations Factorization) } \\
\mathcal{M}^{-1} & =[I-K \psi H] D^{-1}[I-K \psi H]^{T} & &
\end{aligned}
$$

From the above operator formulation, new operator identities may be established which result in the alternative innovations factorization [24, 51]. An object-oriented C++ toolbox also based on these algorithms is described in [31, 32], where also first extensions to sensitivity calculations are described.

\subsection{Muscle Modeling}

Each muscle exhibits some characteristic behavior due to its internal structure. We state the resulting relations and brief explanations of them. The models are widely used and based on the Hill type phenomenological model [43]. A survey of all relations may be found in [55]; the structure itself shall not be discussed here. The following relations hold for each muscle $i, i=1, \ldots, n_{m}$. The index $i$ is omitted for the sake of brevity in the formulae of the following subsections. The values of all parameters and details on the right hand sides of the formulae are given in Section 5.

\subsubsection{Force-Velocity Relation}

The active force a muscle may exert depends on its velocity $v^{M}[62,65,11,35]$. It is equal to the muscle maximum isometric force at zero velocity and equal to zero at the maximum contraction velocity. The active force is higher than the maximum isometric force if the muscle has excentric velocity. The overall relation not only depends on the maximum velocity $v_{m a x}^{M}$ but also on parameters $c_{3}, c_{4}$ that indicate how fast the force converges to zero with contractive velocity resp. how fast the force converges to the maximum force with excentric velocity. For fast muscles $c_{3} \in[0.25,1]$, while for slow muscles, $c_{3} \in[0.1,0.25]$. $c_{4}$ is given by $c_{4}=\frac{-0.33}{2} \frac{c_{3}}{1+c_{3}}$. The overall force-velocity relation is given by:

$$
f_{F V}\left(v^{M}\right)=\left\{\begin{array}{ll}
\frac{1-\frac{v^{M}}{v_{m a x}^{M}}}{1+\frac{M M}{v_{\text {max }}^{M} c_{3}}} & , v^{M} \leq 0 \\
\frac{1-1.33 \frac{v^{M}}{v_{M a x} c_{4}}}{1-\frac{v M}{v_{\text {max }}^{M} c_{4}}} & , v^{M}>0 .
\end{array}, i=1, \ldots, n_{m} .\right.
$$

Figure 1 shows two examples of the force-velocity relation for a fast and a slow muscle.

\subsubsection{Tension-Length Relation}

Muscle forces result from biochemical structures that grip into each other and thereby cause the movement respective force. The more overlapping structures exist, the higher are the forces that may be established. If the muscle is expanded, less overlapping area and thus less force exists. If the muscle on the other hand is shortened, the structures obstruct each other and also less force may be exerted. This property is modeled by the following equations, where $l^{M}$ is the length of the muscle, $l_{0}^{M}$ its rest length, $c_{1}$ and $c_{2}$ are parameters for the effect of decrease of forces when expanding resp. shortening the muscle $[16,48]$. Figure 2 gives an example of the relation.

$$
f_{T L}\left(l^{M}\right)= \begin{cases}e^{-\frac{1}{c_{1}}\left(1-\frac{l^{M}}{1.1 l_{0}^{M}}\right)^{3}} & , l^{M} \leq 1.1 l_{0}^{M} \\ e^{-\frac{1}{c_{2}}\left(\frac{l^{M}}{1.1 l_{0}^{M}}-1\right)^{3}} & , l^{M}>1.1 l_{0}^{M}\end{cases}
$$

\subsubsection{Activation Dynamics}

Muscles may not exert force instantaneously [27]. Muscle excitation $u$ leads to an increased calcium ion concentration $\gamma$ in the muscle which finally results in force exertion. This property is modeled with suitable parameters $b_{1}, b_{2}, b_{3}$ by:

$$
\dot{\gamma}=b_{2}\left(b_{3} u-\gamma\right)
$$



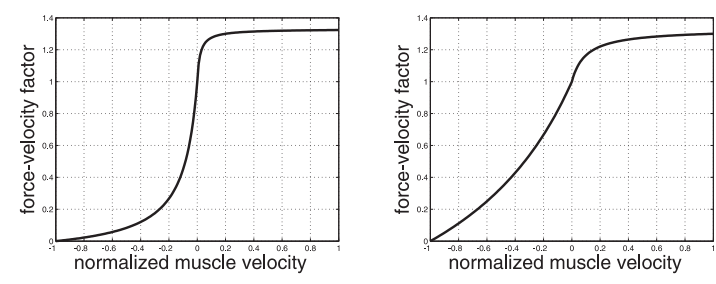

Fig. 1 Force-velocity relation for a slow muscle $\left(c_{3}=\right.$ $0.1, c_{4}=0.02$; left $)$ and for a fast muscle $\left(c_{3}=1.0, c_{4}=0.1\right.$; right).

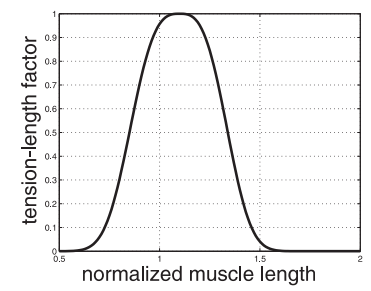

Fig. 2 Tension-length relation for example parameter values $\left(c_{1}=0.017, c_{2}=0.015\right)$

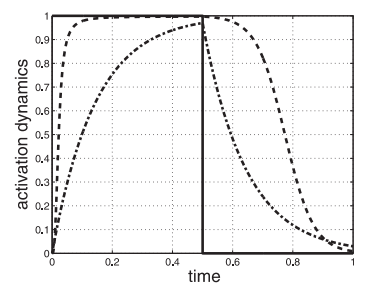

Fig. 3 Muscle activation dynamics (solid line is $u$, dotted line is $\gamma$, dashed line is $f_{A D}$ )

How the calcium ion concentration relates to the force exerted is given by the following equation:

$$
f_{A D}(\gamma(u))=\frac{\left(b_{1} \gamma(u)\right)^{3}}{1+\left(b_{1} \gamma(u)\right)^{3}}
$$

The overall muscle activation dynamics $\left(u, \gamma, f_{A D}\right)$ is shown in Figure 3.

\subsubsection{Muscle Path}

The muscle lengths and velocities needed for the relations above may be expressed by joint angles and angular velocities

$$
l^{M}=l\left(q_{1}, q_{2}, \ldots\right), \quad v^{M}=v\left(q_{1}, q_{2}, \ldots, \dot{q}_{1}, \dot{q}_{2}, \ldots\right) .
$$

To calculate the torques that result from the linear muscle forces, the muscle paths, i.e. the points and directions of application (or the resulting lever directly), have to be modeled. The resulting lever depends on the joint angles only (the first index $i$ indicates the number of the muscle or muscle group, the second index $j$ the number of the joint, the muscle has effects on; not all combinations of $i, j$ are needed):

$$
d_{i, j}=d_{i, j}\left(q_{1}, q_{2}, \ldots\right), \quad i=1, \ldots, n_{m}, j=1, \ldots, n_{q} .
$$

\subsubsection{Elastic and Damping Elements}

Serial elastic and parallel damping elements are described by:

$$
F^{P E E}\left(l^{M}\right)=k_{1}\left(e^{k_{2}\left(l^{M}-k_{3}\right)}-1\right)+k_{4}\left(e^{k_{5} *\left(l^{M}-k_{6}\right)}-1\right) \text { and } F^{D E}\left(v^{M}\right)=k_{0} v^{M} .
$$

\subsubsection{Total Muscle Force}

With the factors given in the previous section and forces generated by parallel elastic elements and damping elements, the total muscle force may be stated as:

$$
F\left(\gamma, l^{M}, v^{M}\right)=F_{\max }^{\text {iso }} f_{A D}(\gamma) f_{T L}\left(l^{M}\right) f_{F V}\left(v^{M}\right)+F^{P E E}\left(l^{M}\right)+F^{D E}\left(v^{M}\right) .
$$

\subsubsection{Resulting Active Torques}

The torque in joint $j$ that results from the applied muscle forces is (with appropriate index sets $I_{j}$ that indicate which muscles have effect on joint $j$ ):

$$
\tau_{j, a}=\sum_{i \in I_{j}} d_{i, j} F_{i}\left(\gamma_{i}, l_{i}^{M}, v_{i}^{M}\right), j=1, \ldots, n_{q} .
$$

\subsubsection{Passive Torques}

In addition to the active torques, passive torques that depend on $\boldsymbol{l}^{M}, \boldsymbol{v}^{M}, \boldsymbol{\gamma}$ (bold letters indicate the vector of all occurring lengths, velocities, calcium ion concentrations), and the joint angles have to be considered [30,67]. These model passive effects of tendons, ligament and the connective tissue (especially at the boundaries of the feasible joint angle intervals)

$$
\tau_{j, p}=\tau_{j, p}\left(\boldsymbol{l}^{\boldsymbol{M}}, \boldsymbol{v}^{\boldsymbol{M}}, \boldsymbol{\gamma}, \boldsymbol{q}\right)
$$

The total torque applied to joint $j$ is $\tau_{j}=\tau_{j, a}+\tau_{j, p}$. It should be noted that for robotic systems $\boldsymbol{u}$ usually describes the torques in the actuated joints which are equal to the control in the optimal control problem if no detailed motor model is used. For biomechanic systems $\boldsymbol{u}$ denotes the controls (i.e. the muscle activations) and $\boldsymbol{\tau}=\left(\tau_{1}, \tau_{2}, \ldots\right)$ are the torques for the dynamics calculations. 


\section{A New Approach to Efficient Forward Dynamics Simulation and Optimization}

To improve the computational cost of forward dynamics simulation by two orders of magnitude we suggest a new approach based on a combination of tailored MBS dynamics modeling and numerical optimal control methods.

\subsection{Formulation of the Forward Dynamics Optimization Problem}

In the biomechanics forward dynamics optimization problem, the muscle activations $\boldsymbol{u}=\left(u_{1}, \ldots, u_{n_{m}}\right)^{T}$ are to be determined so that for goal oriented motion the objective function $\mathcal{J}$ is minimized (e.g. $J=t_{f}$ for minimum time), whereas for analysis of measured motion $\boldsymbol{q}_{\boldsymbol{m}}(t), 0 \leq t \leq t_{f}$, the objective function usually is chosen to be $\mathcal{J}=\int_{0}^{t_{f}}\left(\boldsymbol{q}(t)-\boldsymbol{q}_{\boldsymbol{m}}(t)\right)^{2} d t$. One summand for distributing load to the muscles involved, cf. Subsection 2.2.3, may be introduced as well.

The biomechanical multibody system is constrained to behave according to the MBS differential equation (2). The total torque $\tau$ consists of passive and active torques, cf. Subections 3.2.8 and 3.2.7. In addition to the MBS ODEs which are computed using a recursive ABA method (cf. Section 3.1), the differential equations for activation dynamics (cf. Subsection 3.2.3) must be included in the problem formulation. Boundary constraints, e.g. for initial or final position, and nonlinear state and control constraints, e.g. for geometric constraints, depend on the motion to be computed.

\subsection{General Optimal Control Problem}

The differential equations of motion of second order are transformed into a set of double size of differential equations of first order. The vector $\boldsymbol{x}$ of state variables then is $\boldsymbol{x}=\left(q_{1}, \ldots, q_{n_{q}}, \dot{q}_{1}, \ldots, \dot{q}_{n_{q}}, \gamma_{1}, \ldots, \gamma_{n_{m}}\right)^{T}$, where $q_{i}, \dot{q}_{i}$ are the joint angles and velocities of joint $i, \gamma_{j}$ are the $\mathrm{ca}^{2+}$ ion concentration in muscle $j, n_{q}$ is the number of joints and $n_{m}$ is the number of muscles or muscle groups. The vector $\boldsymbol{u}$ of controls is $\boldsymbol{u}=\left(u_{1}, \ldots, u_{n_{m}}\right)^{T}$, where $u_{j}$ is the activation of muscle (group) $j$.

Thus, the biomechanics optimal control problem may be stated in the following form of a general constrained optimal control problem for state differential equations of first order:

$$
\begin{array}{ll}
\min \boldsymbol{u} \mathcal{J}=\min \boldsymbol{u}\left(\varphi\left(\boldsymbol{x}\left(t_{f}\right), \boldsymbol{x}, t_{f}\right)+\int_{0}^{t_{f}} L(\boldsymbol{x}(t), \boldsymbol{u}(t), \boldsymbol{p}, t) d t\right) & \begin{array}{l}
\text { Minimize the objective function } \mathcal{J} \text { consisting } \\
\text { of Mayer (scalar) and Lagrange (integral) term }
\end{array} \\
\text { subject to } & \\
\dot{\boldsymbol{x}}(t)=\boldsymbol{f}(\boldsymbol{x}(t), \boldsymbol{u}(t), \boldsymbol{p}, t) & \text { system of ordinary differential equations } \\
\boldsymbol{r}\left(\boldsymbol{x}(0), \boldsymbol{x}\left(t_{f}\right), \boldsymbol{p}, t_{f}\right)=0 & \text { boundary constraints } \\
g_{i}(\boldsymbol{x}(t), \boldsymbol{u}(t), \boldsymbol{p}, t) \geq 0, i=1, \ldots, n_{g}, 0 \leq t \leq t_{f} & \text { nonlinear state and control constraints }
\end{array}
$$

where $\boldsymbol{x}=\left(x_{1}, \ldots, x_{n_{x}}\right)^{T}$ are the states, $\boldsymbol{u}=\left(u_{1}, \ldots, u_{n}\right)^{T}$ are the controls and $\boldsymbol{p}$ are constant (but to be optimized) parameters.

\subsection{Direct Collocation Method}

There are many different approaches for solving optimal control problems. Here, we consider the computation of optimal trajectories $\boldsymbol{x}^{*}, \boldsymbol{u}^{*}$ subject to a large and highly nonlinear dynamical system. For this class of problems, so-called direct (transcription) methods have been developed in recent years showing remarkable performance [7, 64]. Instead of using one of the direct shooting approaches mentioned in Section 2.3.2 which require feasibility with respect to the ODE constraints in each iteration of the optimization method a simultaneous approach for solving the ODE integration and optimization problems inherent in the optimal control problem is selected. In direct collocation the implicit integration for a sequence of steps from initial to final time is included as a set of explicit nonlinear equality constraints in the optimization problem. Without the restriction to feasibility to the ODE constraints in each iteration as in direct shooting only the final solution of direct collocation iteration must satisfy them. Without the restriction of feasible iterates and with much easier computable gradients the solution may be obtained much faster.

The direct collocation method DIRCOL [63] is based on the discretization of both the states and the controls, i.e. $\boldsymbol{x}(t)$ and $\boldsymbol{u}(t)$ are approximated by $\tilde{\boldsymbol{x}}(t)$ and $\tilde{\boldsymbol{u}}(t)$ on a grid $0=t_{1}<t_{1}<\ldots<t_{n_{t}}=t_{f}$ :

$$
\tilde{\boldsymbol{x}}(t)=\sum_{l} \alpha_{l} \hat{\boldsymbol{x}}_{l}(t), \tilde{\boldsymbol{x}} \in S_{\Delta}^{4} \text { (cubic), } \quad \tilde{\boldsymbol{u}}(t)=\sum_{j} \beta_{j} \hat{\boldsymbol{u}}_{j}(t), \tilde{\boldsymbol{u}} \in S_{\Delta}^{2} \text { (linear), }
$$

where $\hat{\boldsymbol{x}}_{l}, \hat{\boldsymbol{u}}_{j}$ are basis functions (e.g. monomials or Hermite basis functions). $\alpha_{l}, \beta_{j}$ are the coefficients of the piecewise polynomial approximation of the states resp. controls and become the variables in the resulting nonlinear constrained optimization problem (NLP). Thus, the resulting large-scale NLP becomes:

$$
\boldsymbol{y}=\left(\alpha_{1}, \alpha_{2}, \ldots, \beta_{1}, \beta_{2}, \ldots, t_{n_{t}}\right)^{T}, \quad \quad \min _{\boldsymbol{y}} \phi(\boldsymbol{y}) \text { s.t. } \boldsymbol{a}(\boldsymbol{y})=0, \boldsymbol{b}(\boldsymbol{y}) \geq 0
$$

where the equality and inequality constraints $\boldsymbol{a}$ and $\boldsymbol{b}$ are the following: 


$$
\begin{array}{ll}
\dot{\tilde{\boldsymbol{x}}}\left(t_{\diamond}\right)-f\left(\tilde{\boldsymbol{x}}\left(t_{\diamond}\right), \tilde{\boldsymbol{u}}\left(t_{\diamond}\right), t_{\diamond}\right)=0, t_{\diamond}=t_{k}, t_{k+1 / 2}, t_{k+1} & \text { collocation constraints at the grid points } \\
r\left(\dot{\tilde{\boldsymbol{x}}}\left(t_{1}\right), \dot{\tilde{\boldsymbol{x}}}\left(t_{n_{t}}, \boldsymbol{p}, t_{n_{t}}\right)\right)=0 & \text { and the midpoints of all intervals }\left[t_{i}, t_{i+1}\right. \\
g_{i}\left(\tilde{\boldsymbol{x}}\left(t_{k}\right), \tilde{\boldsymbol{u}}\left(t_{k}\right), \boldsymbol{p}, t_{k}\right) \geq 0, i=1, \ldots, n_{g}, k=1, \ldots, n_{t} & \text { inequality constraints. }
\end{array}
$$

By solving the NLP, the differential equations of motion are solved simultaneously with the optimization problem. This leads to a considerable improvement of efficiency compared to standard methods if all structure and sparsity in the NLP is utilized using a sparse sequential quadratic programming method of [19]. The time grid is refined based on local error estimates resulting in a sequence of NLPs with increasing dimensions which are solved successively.

\section{Numerical Results: Kicking Motion}

A time optimal kicking movement has been investigated [60,61]. Kinematic and kinetic data of the musculoskeletal system as well as muscle model parameters and measured reference data have been taken from Spägele [55, 58]. The model (cf. Figure 6) consists of two joints, two rigid links and five muscle groups. The problem is formulated as an optimal control problem with 9 states (hip angle $q_{1}$, knee angle $q_{2}$, the corresponding joint velocities and 5 calcium ion concentrations) and 5 controls (activations of the muscles). The kicking movement was optimized to be time optimal, i.e. the objective function is $\mathcal{J}=t_{f}$. The muscle lengths (cf. Equation 8, subscript here denotes the number of the muscle) are calculated according to [55]

$$
\begin{aligned}
& l_{1}^{M}=0.287-0.0497 q_{1} \\
& l_{2}^{M}=0.300+0.0330 q_{2} \\
& l_{3}^{M}=0.517+0.045 \cos \left(1.128 q_{1}+0.748\right)+0.033 q_{2} \\
& l_{4}^{M}=0.483-0.062 \cos \left(1.047 q_{1}+0.838\right)+0.07 \cos \left(1.076 q_{2}+0.28\right) \\
& l_{5}^{M}=0.088+0.019 \cos \left(1.16 q_{2}+0.464\right)
\end{aligned}
$$

The velocities are the time derivatives of the lengths, $v_{i}^{M}=i_{i}^{M}, i=1, \ldots, 5$. The resulting lever arms (Equation 9) are also taken from [55]:

$$
\begin{aligned}
& d_{1, q_{1}}=0.024+0.0188 q_{1} \\
& d_{2, q_{2}}=0.036+0.03 e^{-4.33\left(0.17-q_{2}\right)^{2}} \\
& d_{3, q_{1}}=0.052 \cos \left(q_{1}-0.63\right)-0.002 \\
& d_{4, q_{1}}=0.037 \cos \left(1.309 q_{1}-0.916\right)+0.026 \\
& d_{4, q_{2}}=0.058\left(q_{2}+0.685\right)^{2} e^{-1.187 q_{2}} \\
& d_{5, q_{2}}=0.055 .
\end{aligned}
$$

The passive moments (Equation 13) are stated in [55] to be:

$$
\begin{aligned}
& \tau_{1, p}=0.8 e^{-3.41 q_{1}}+0.084 e^{-15 q_{1}}-0.753 e^{2.55 q_{1}}-\left(7.9 e^{-2.72 q_{1}}+0.09 e^{1.8 q_{1}}\right) \dot{q}_{1} \\
& \tau_{2, p}=1.25 \cdot 10^{-7} e^{8.5 q_{2}}-6.3 e^{-2.9 q_{2}}-20.1 e^{-16.1 q_{2}}+2.1-\left(0.3 e^{1.02 q_{2}}+1.85 e^{-3.43 q_{2}}\right) \dot{q}_{2} .
\end{aligned}
$$

The multibody system parameters (mass, inertia w.r.t. point of rotation, center of mass, length) for the thigh and shank are

$$
m_{1}=8.692, I_{1}=0.480, z_{1}=0.189, l_{1}=0.447, m_{2}=15.492, I_{2}=4.700, z_{2}=0.501, l_{2}=0.538 .
$$

All other parameters may be found in Figure 5. The boundary conditions were set to

$$
\begin{aligned}
& q_{1}\left(t_{0}\right)=0.1, q_{2}\left(t_{0}\right)=0.15, \dot{q}_{1}\left(t_{0}\right)=\dot{q}_{2}\left(t_{0}\right)=0, \gamma_{1}\left(t_{0}\right)=\ldots=\gamma_{t}\left(t_{0}\right)=0 \\
& q_{1}\left(t_{f}\right)=0.8, q_{2}\left(t_{f}\right)=-0.05, \dot{q}_{2}\left(t_{f}\right)=0 .
\end{aligned}
$$

Box constraints are imposed on the states and controls

$$
0 \leq q_{1} \leq 1.5,-0.05 \leq q_{2} \leq 1.5,0 \leq u_{i}, \gamma_{i} \leq 1, i=1, \ldots, 5
$$

An overview of the data flow in the kicking model may be found in Figure 4.

Compared to the measured movements (and the results of $[55,58]$, which match the measured data very well), our results show a shorter time and higher maximum angles (cf. Figure 8). The reason for this is, that in [55] the maximum muscle forces were modified to match the optimized time of the measurement. Obviously our optimal movement is another local minimum. Nevertheless, the controls (Figure 9) show the same characteristics. Computing time and size of the resulting NLP are shown in Figure 7. The direct shooting approach used in [55, 58] for 11 grid points required hours to compute the solution [57]. Comparing the computing time with our approach (Figure 7) and considering how computational speed has progressed since 1996, we still obtain a speed up of two orders of magnitude. 


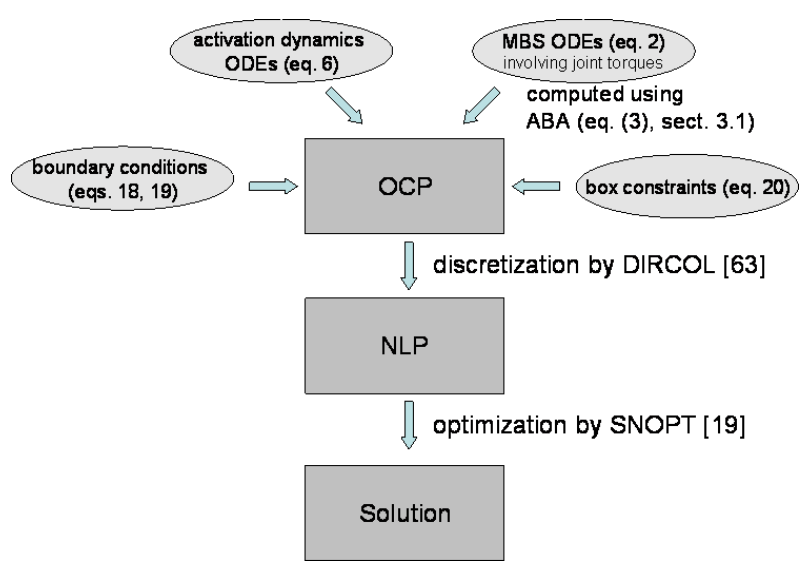

Fig. 4 Schematic summary of the method.

1. Ilio Psoas group

2. Vastus group

3. Rectus Femoris

4. Hamstring group

5. Gastrocnemius group

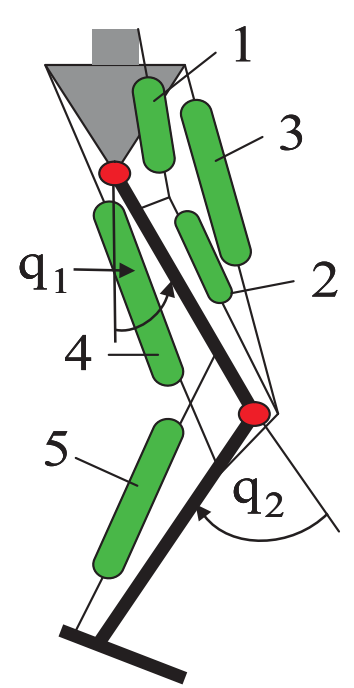

Fig. 6 Kinematic structure of the leg with 5 muscle groups.

\begin{tabular}{|l||c|c|c|c|c|}
\hline parameter & muscle 1 & muscle 2 & muscle 3 & muscle 4 & muscle 5 \\
\hline$b_{1}$ & 7.23 & 7.23 & 7.23 & 7.23 & 7.23 \\
\hline$b_{2}$ & 7.46 & 7.61 & 8.37 & 7.46 & 7.61 \\
\hline$b_{3}$ & 1.0 & 1.0 & 1.0 & 1.0 & 1.0 \\
\hline$k_{0}[N]$ & 0.0 & 0.0 & 257.1 & 378.0 & 0.0 \\
\hline$k_{1}[N]$ & 0.0 & 0.0 & 5.393 & 64.7 & 0.0 \\
\hline$k_{2}\left[\mathrm{~m}^{-1}\right]$ & 0.0 & 0.0 & 90.4 & 23.95 & 0.0 \\
\hline$k_{3}[\mathrm{~m}]$ & 0.0 & 0.0 & 0.58 & 0.48 & 0.0 \\
\hline$k_{4}[N]$ & 0.0 & 0.0 & 0.0 & 0.0068 & 0.0 \\
\hline$k_{5}\left[\mathrm{~m}^{-1}\right]$ & 0.0 & 0.0 & 0.0 & 239.8 & 0.0 \\
\hline$k_{6}[\mathrm{~m}]$ & 0.0 & 0.0 & 0.0 & 0.53 & 0.0 \\
\hline$c_{1}$ & 0.017 & 0.017 & 0.017 & 0.017 & 0.017 \\
\hline$c_{2}$ & 0.015 & 0.015 & 0.015 & 0.015 & 0.015 \\
\hline$l_{0}^{M}[\mathrm{~m}]$ & 0.258 & 0.309 & 0.500 & 0.486 & 0.085 \\
\hline$c_{3}$ & 0.50 & 0.33 & 0.33 & 0.50 & 0.50 \\
\hline$c_{4}$ & 0.09 & 0.02 & 0.08 & 0.10 & 0.03 \\
\hline$v_{\max }^{M}[\mathrm{~m} / \mathrm{s}]$ & -1.6 & -0.5 & -2.0 & -1.8 & -0.5 \\
\hline$F_{\max }^{\text {1so }}[\mathrm{N}]$ & 4800.0 & 5300.0 & 1200.0 & 1500.0 & 700.0 \\
\hline
\end{tabular}

Fig. 5 Parameters for Equations (4), (5), (6), (7), (10) of the kicking model (taken from [55]).

\begin{tabular}{|l|l|l|}
\hline grid points & 10 & 60 \\
\hline nonlinear constraints & 81 & 829 \\
\hline nonlinear variables & 129 & 531 \\
\hline computing time & $1.2 \mathrm{~s}$ & $6.3 \mathrm{~s}$ \\
\hline
\end{tabular}

Fig. 7 Size of the resulting NLP and computation time on a 1700 $\mathrm{MHZ}+$ Athlon XP for two different numbers of grid points in the discretization.
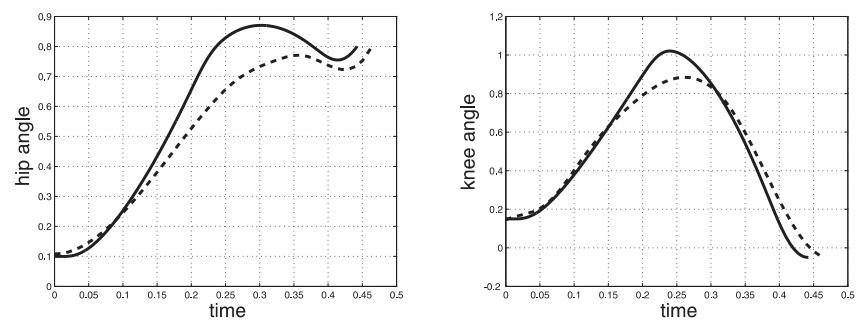

Fig. 8 Measured (dashed line) and optimized (solid line) joint angle trajectory of hip (left) and knee (right).
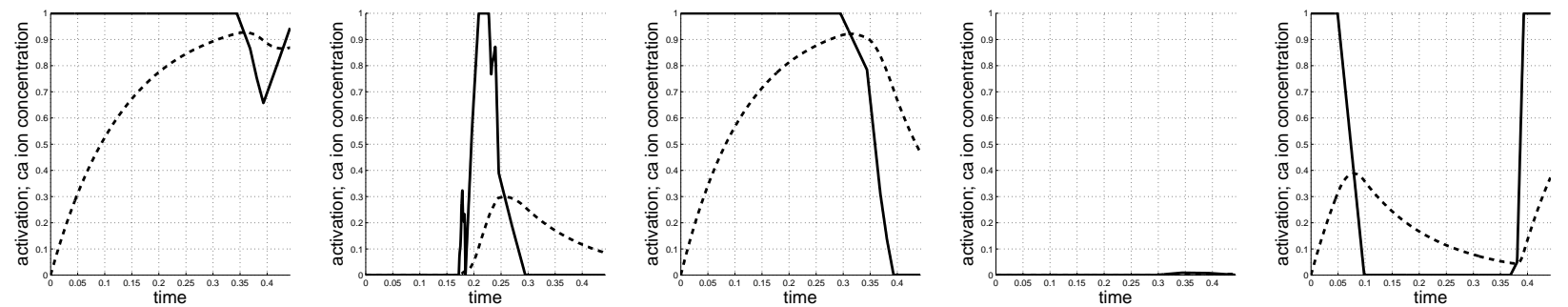

Fig. 9 Results from optimization: Controls (corresponding to EMG, solid line) and calcium ions concentrations (dashed line).

\section{Conclusions and Outlook}

First steps towards a new approach to solving forward dynamics simulation and optimization of human body dynamics very efficiently have been presented. It is based on an efficient, recursive modeling of human motion dynamics and a tailored numerical optimal control method. First numerical results show an improvement in computational cost of two orders of magnitude.

However, many issues are still open and need to be addressed: The problem of whole human body dynamics motions with multiple contacts, like it occurs for javelin throw or gymnastics, needs further development: contact models must be investigated and brought to a form applicable to the presented approach. Another important issue in human body dynamics simulation is data collection. For the example kicking motion, the data has been taken from literature. One difficult issue 
is that not only motion data (joint angles and velocities and contact forces) from one person's motion are needed but also the kinetic, anthropometric data (MBS data, muscle data) of the same person.

Ongoing research includes the investigation of several other examples of human motion, the incorporation of other objective functions, wobbling masses, a refined foot model, investigations on the fatigue of muscles, the extension of the human body dynamics modeling algorithms to the computation of Jacobians and a further improved adaption of the optimal control method to utilize the special structure of first and second order differential equations of motion. In cooperation with the Institute of Ergonomics of the Technische Universität Darmstadt, measurement data of human motion is being collected for these investigations.

Acknowledgements The research presented in this paper was supported by the German Research Foundation DFG under grant STR 533/3-1. The authors thank Dr. Thomas Spägele for making many data available to them.

\section{References}

[1] URL: http://www.nlm.nih.gov/research/visible.

[2] F. C. Anderson and M. G. Pandy. A dynamic optimization solution for vertical jumping in three dimensions. Comp. Meth. Biomech. Biomed. Eng., 2:201-231, 1999.

[3] F. C. Anderson and M. G. Pandy. Dynamic optimization of human walking. J. of Biomechanic Engineering, 123:381-390, 2001.

[4] F. C. Anderson and M. G. Pandy. Static and dynamic optimization solutions for gait are practically equivalent. Journal of Biomechanics, 34:153-161, 2001.

[5] F. C. Anderson, J. Ziegler, M. G. Pandy, and R. T. Whalen. Application of high-performance computing to numerical simulation of human movement. J. of Biomechanic Engineering, 117:155-157, 1995.

[6] T. Angeli, M. Gföhler, T. Eberharter, P. Lugner, L. Rinder, and H. Kern. Optimization of the pedal path for cycling powered by lower extremity muscles activated by functional electrical stimulation. In J. Middleton, M. Jones, N. Shrive, and G. Pande, editors, Computer Methods in Biomechanics and Biomedical Engineering-3. Gordon and Breach, 2001.

[7] J. Betts. Practical Methods for Optimal Control Using Nonlinear Programming. SIAM, 2001.

[8] M. Damsgaard, S. Christensen, and J. Rasmussen. An efficient numerical algorithm for solving the muscle recruitment problem in inverse dynamics simulations. In Intern. Society of Biomechanics, XVIIIth Congress, July 8-13, 2001, Zurich, Switzerland, 2001.

[9] P. Eberhard, T. Spägele, and A. Gollhofer. Investigations for the dynamical analysis of human motions. Multibody System Dynamics, 3:1-20, 1999.

[10] R. Featherstone. Robot Dynamics Algorithms. Kluwer Academic Publishers, 1987.

[11] Y. C. Fung. Biomechanics. Springer-Verlag, New York, 1981.

[12] B. A. Garner and M. G. Pandy. A kinematic model of the uppe limb based on the visiblie human project (vhp) image dataset. Computer Methods in Biomechnics and Biomedical Engineering, 2:107-124, 1999.

[13] B. A. Garner and M. G. Pandy. The obstacle-set method for representing muscle paths in musculoskeletal models. Computer Methods in Biomechanics and Biomedical Engineering, 3:1-30, 2000.

[14] B. A. Garner and M. G. Pandy. Musculoskeletal model of the upper limb based on the visible human male dataset. Computer Methods in Biomechanics and Biomedical Engineering, 4:93-126, 2001.

[15] B. A. Garner and M. G. Pandy. Estimation of musculotendon properties in the human upper lim. Annals of Biomedical Engineering, 31:207-220, 2003.

[16] H. S. Gasser and A. V. Hill. The dynamics of muscular contraction. Proceedings of the Royal Society of London, 96 B:398-437, 1924.

[17] M. Gföhler, T. Angeli, and P. Lugner. Optimal control of cycling by means of functional electrical stimulation - a dynamic simulation study. In VIII International Symposium on Computer Simulation in Biomechanics July 4th-6th 2001, Milan, Italy, 2001.

[18] L. Gilchrist and D. Winter. A two-part, viscoelastic foot model for use in gait simulations. J. Biomechanics, 29(6):795-798, 1996.

[19] P. Gill, W. Murray, and M. Saunders. SNOPT: An SQP algorithm for large-scale constrained optimization. SIAM Journal on Optimization, 12:979-1006, 2002.

[20] K. Gruber. Entwicklung eines Modells zur Berechnung der Kräfte im Knie- und Hüftgelenk bei sportlichen Bewegungsabläufen mit hohen Beschleunigungen. PhD thesis, Eberhard-Karls-Universität, Tübingen, 1987.

[21] K. Gruber, H. Ruder, J. Denoth, and K. Schneider. A comparative study of impact dynamics. wobbling mass model versus rigid body models. Journal of Biomechanics, 31:439-444, 1998.

[22] M. Günther. Computersimulation zur Synthetisierung des muskulär erzeugten menschlichen Gehens unter Verwendung eines biomechanischen Mehrkörpermodells. PhD thesis, Eberhard-Karls-Universität zu Tübingen, 1997.

[23] R. Happee. Inverse dynamic optimization including muscular dynamics, a new simulation method applied to goal directed movements. Journal of Biomechanics, 27:953-960, 1994.

[24] M. Hardt. Multibody Dynamical Algorithms, Numerical Optimal Control, with Detailed Studies in the Control of Jet Engine Compressors and Biped Walking. PhD thesis, Electrical Engineering, University of California San Diego, U.S.A., 1999.

[25] M. W. Hardt and O. von Stryk. Dynamic modeling in the simulation, optimization, and control of bipedal and quadrupedal robots. Z. Angew. Math. Mech., 83(10):648-662, 2003.

[26] H. Hatze. The complete optimization of a human motion. Mathematical Biosciences, 28:99-135, 1976.

[27] H. Hatze. Myocybernetic control models of skeletal muscle. Gutenberg Book Printers, Pretoria, 1981.

[28] E. Haug. Computer aided kinematics and dynamics of mechanical systems: Basic Methods. Allyn and Bacon, Boston, 1989. 
[29] A. Henze. Dreidimensionale biomechanische Modellierung und die Entwicklung eines Reglers zur Simulation zweibeinigen Gehens. $\mathrm{PhD}$ thesis, Eberhard-Karls-Universität zu Tübingen, 2002.

[30] A. L. Hof and J. van den Berg. Emg to force processing ii: estimation of parameters of the hill muscle model for the human triceps surae by means of a calferometer. Journal of Biomechanics, 14:759-770, 1981.

[31] R. Höpler. A unifying object-oriented methodology to consolidate multibody dynamics computations in robot control. $\mathrm{PhD}$ thesis, Technische Universität Darmstadt, 2004.

[32] R. Höpler, M. Stelzer, and O. von Stryk. Object-oriented dynamics modeling for legged robot trajectory optimization and control. In Proc. IEEE Conf. on Mechatronics and Robotics (MechRob), pages 972-977), Aachen, September 13-15 2004.

[33] A. Jain. Unified formulation of dynamics for serial rigid multibody systems. 14(3):531-542, 1991.

[34] M. L. Kaplan and J. H. Heegaard. Predictive algorithms for neuromuscular control of human locomotion. Journal of Biomechanics, 34(8):1077-1083, 2001.

[35] B. Katz. The relation between force and speed in muscular contraction. Journal of Physiology, 96:45-64, 1939.

[36] M. Kiehl. Sensitivity analysis of ODEs and DAEs - theory and implementation guide. Optimization Methods and Software, 10(6):803-821, 1999.

[37] W. Liu and B. M.Nigg. A mechanical model to determine the influence of masses and mass distribution on the impact force during running. Journal of Biomechanics, 33:219-224, 2000.

[38] S. McMillan and D. Orin. Forward dynamics of multilegged vehicles using the composite rigid body method. In IEEE International Conference on Robotics and Automation, pages 464-470, 1998.

[39] Y. Nakamura, K. Yamane, I. Suzuki, and Y. Fujita. Dynamic computation of musculo-skeletal human model based on efficient algorithm for closed kinematic chains. Proceedings of the 2nd International Symposium on Adaptive Motion of Animals and Machines, Kyoto, March 4-8, 2003, 2003.

[40] R. R. Neptune and M. L. Hull. Evaluation of performance criteria for simulation of submaximal steady-state cycling using a forward dynamic model. Journal of Biomechanical Engineering, 120:334-340, 1998.

[41] R. R. Neptune and M. L. Hull. A theoretical analysis of preferred pedaling rate selection in endurance cycling. Journal of Biomechanics, 32:409-415, 1999.

[42] R. R. Neptune, I. C. Wright, and A. J. Van den Bogert. A method for numerical simulation of single limb ground contact events: application to heel-toe running. Computer Methods in Biomechanics and Biomedical Engineering, 3:321-344, 2000.

[43] B. M. Nigg and W. Herzog. Biomechanics of the Musculo-skeletal System. Wiley, 1999.

[44] D. Pai, U. Ascher, and P. Kry. Forward dynamics algorithms for multibody chains and contact. In IEEE International Conference on Robotics and Automation, pages 857-863, 2000.

[45] M. G. Pandy. Computer modeling and simulation of human movement. Annu. Rev. Biomed. Eng., 3:245-273, 2001.

[46] M. G. Pandy, F. C. Anderson, and D. G. Hull. A parameter optimization approach for the optimal control of large-scale musculoskeletal systems. Journal of Biomechanical Engineering, 114:450-460, 1992.

[47] M. G. Pandy and K. Sasaki. A three-dimensional musculoskeletal model of the human knee joint. part 2: Analysis of ligament function. Computer Methods in Biomechnis and Biomedial Engineering, 1:265-283, 1998.

[48] R. W. Ramsey and S. F. Street. The isometric length-tension diagram of isolated skeletal muscle fibres of the frog. Journal of Cellular and Comparative Physiology, 15:11-34, 1940.

[49] J. Rasmussen, M. Damsgaard, and S. T. Christensen. Optimization of human motion: to invert inverse dynamics. International Society of Biomechanics, XVIIIth Congress, July 8-13, 2001, Zurich, Switzerland, 2001.

[50] J. Rasmussen, M. Damsgaard, and M. Voigt. Muscle recruitment by the min/max criterion? a comparative numerical study. Journal of Biomechanics, 34(3):409-415, 2001.

[51] G. Rodriguez, K. Kreutz-Delgado, and A. Jain. A spatial operator algebra for manipulator modeling and control. International Journal of Robotics Research, 40:21-50, 1991.

[52] W. Schiehlen, editor. Multibody Systems Handbook. Springer-Verlag, 1990.

[53] W. Schiehlen, editor. Advanced Multibody System Dynamics: Simulation and Software Tools. Kluwer, 1993.

[54] A. Seyfarth, A. Friedrichs, V. Wank, and R. Blickhan. Dynamics of the long jump. Journal of Biomechanics, 32:1259-1267, 1999.

[55] T. Spägele. Modellierung, Simulation und Optimierung menschlicher Bewegungen. PhD thesis, Universität Stuttgart, 1998.

[56] T. Spägele. Personal communication. 2002.

[57] T. Spägele. personal communication. 2005.

[58] T. Spägele, A. Kistner, and A. Gollhofer. Modelling, simulation and optimisation of a human vertical jump. Journal of Biomechanics, 32(5):521-530, 1999.

[59] T. Spägele, A. Kistner, and A. Gollhofer. A multi-phase optimal control technique for the simulation of a human vertical jump. Journal of Biomechanics, 32(1):89-91, 1999.

[60] M. Stelzer and O. von Stryk. Efficient forward dynamics simulation and optimization of locomotion: from legged robots to biomechanical systems. In Proc. 3rd Intl. Symposium on Adaptive Motion in Animals and Machines (AMAM), 2005.

[61] M. Stelzer and O. von Stryk. From robots to humans: Towards efficient forward dynamics simulation and optimization. In Proc. of ECCOMAS Multibody Dynamics 2005, Advances in Computational Multibody Dynamics, Madrid, June 21-24, 2005.

[62] A. J. van Soest. Jumping from structure to control. Phd thesis, Vrije Universiteit Amsterdam, 1992.

[63] O. von Stryk. Numerical Hybrid Optimal Control and Related Topics. Habilitationsschrift, Technische Universität München, 2003.

[64] O. von Stryk and R. Bulirsch. Direct and indirect methods for trajectory optimization. Annals of Operations Research, 37:357-373, 1992.

[65] D. R. Wilkie. The mechanical properties of muscle. British Medical Bulletin.

[66] R. C. Woledge, N. A. Curtin, and E. L. Homsher. Energetic aspects of muscle contraction. Academic Press, 1985.

[67] Y. S. Yoon and J. M. Mansour. The passive elastic moment at the hip. Journal of Biomechanics, 15:905-910, 1982.

[68] D. Zlatnik. Intelligently Controlled Above Knee Prosthesis. PhD thesis, ETH Zürich, 1998. 